\title{
HALF-INTEGRAL WEIGHT MODULAR FORMS AND REAL QUADRATIC $p$-RATIONAL FIELDS
}

\author{
JILALI ASSIM ${ }^{(1)}$, ZAKARIAE BOUAZZAOUI $^{(2)}$
}

\begin{abstract}
Using half-integral weight modular forms we give a criterion for the existence of real quadratic $p$-rational fields. For $p=5$ we prove the existence of infinitely many real quadratic $p$-rational fields.
\end{abstract}

\section{INTRODUCTION}

The Dedekind zeta function of an algebraic number field encodes a lot of arithmetic information of the field. For a number field $F$, let $\mathcal{O}_{F}$ denote the ring of its integers. For each integer $m$, let $\zeta_{F}^{*}(m)$ denote the leading non-zero coefficient in the Taylor expansion of the Dedekind zeta function of $F$. Dirichlet's class number formula reads:

$$
\zeta_{F}^{*}(0)=-\frac{h_{F}}{w_{F}} \cdot R_{F},
$$

where $h_{F}$ is the class number of $F, w_{F}$ is the number of roots of unity in $F$ and $R_{F}$ is the Dirichlet regulator. We are interested with the divisibility, by odd prime numbers $p$, of the special values of Dedekind zeta functions of real quadratic fields at odd negative integers, these values are closely related to the orders of certain cohomology groups.

Let $S$ be a finite set of primes. Denote by $F_{S}$ the maximal pro- $p$-extension of $F$ which is unramified outside $S$ and let $G_{S}(F)$ be its Galois group. The field $F$ is called $p$-rational if the Galois group $G_{S_{p}}(F)$ of the extension $F_{S_{p}} / F$ is pro-p-free (with rank $1+r_{2}, r_{2}$ being the number of complex primes of $F$ ), where $S_{p}$ is the set of primes of $F$ above $p$. If $F$ is totally real, we prove in section 2 that $F$ is $p$-rational precisely when $v_{p}\left(\zeta_{F}(2-p)\right)=-1$, where $v_{p}$ denotes the $p$-adic valuation. We use this characterization to study $p$-rationality of real quadratic fields. The notion of $p$-rational fields has been introduced to construct extensions of $\mathbf{Q}$ satisfying the Leopoldt conjecture [M-N]. Recently, R. Greenberg [G] used $p$-rational number fields to construct (in a non geometric manner) Galois representations with open image in $\mathrm{GL}_{n}\left(\mathbf{Z}_{p}\right)$ for $n \geq 3$. This paper is motivated by the study of $p$-rationality of multi-quadratic number fields, for which Greenberg formulated the following conjecture:

Conjecture 1. ([G, Conjecture 4.2.1]) For any odd prime number $p$ and any integer $t \geq 1$, there is a p-rational field $F$ such that $\operatorname{Gal}(F / \mathbf{Q}) \cong(\mathbf{Z} / 2 \mathbf{Z})^{t}$.

The conjecture is true for $t=1$, since for every odd prime number $p$, there is infinitely many $p$-rational imaginary quadratic fields (cf. [G, Proposition 4.1.1]). The case $t \geq 2$ leads to the study of $p$-rationality of real quadratic fields, which is the aim of this paper. After relating the $p$-rationality to special values of $L$-functions, we use the theory of modular forms to obtain our results. Roughly speaking, we use Cohen-Eisenstein series [C], which are modular forms of half integer weight, and whose Fourier coefficients involve special values of $L$-functions of quadratic fields. Multiplying such modular forms by theta series produces integer weight modular forms, and the resulting Fourier coefficients are studied to deduce divisibility properties of values of $L$-functions. As a consequence we give for $p=5$ the

2010 Mathematics Subject Classification. 11R11, 11F37, 11R42.

Key words and phrases. L-functions, $p$-rational field, modular forms. 
existence of infinitely many real quadratic 5-rational fields, a similar result for $p=3$ was given implicitly by D. Byeon in [By] using the same techniques.

Theorem 1.1. There are infinitely many fundamental discriminants $d>0$ such that $\mathbf{Q}(\sqrt{d})$ is 5-rational.

The study of $p$-rationality of real quadratic fields is more subtle than the study of $p$ rationality of imaginary quadratic fields, because of complications due to the existence of nontrivial units. Using Cohen-Eisenstein series, Theorem 1.2 below gives a sufficient condition for the existence of a real quadratic $p$-rational field, with some arithmetic properties, for every prime number $p \geq 5$. More precisely, let $f=\sum_{n \geq 0} a(n) q^{n}$ be an integer weight modular form for the congruence subgroup $\Gamma(N), N \geq 1$, with coefficients in the ring of integers of a number field. By a result of Serre [S76, page 20-19], there is a set of primes $\ell \equiv 1\left(\bmod N p^{2}\right)$ of positive density for which

$$
f \mid T(\ell) \equiv 2 f \quad\left(\bmod p^{2}\right),
$$

where $T(\ell)$ denotes the Hecke operator associated to the prime number $\ell$ [Ko, page.153].

Let $\mathcal{L}=\left\{\ell_{1}, \ldots, \ell_{s}\right\}$ be a finite set of odd primes. For every positive square free integer $t$, let $f$ be an element of the space $M_{p}\left(\Gamma_{1}\left(4 p^{2} t \prod_{i=1}^{s} \ell_{i}^{4}\right)\right)$, obtained by multiplication of half integer weight modular forms (Cohen-Eisenstein series and theta series). Denote by $\mathcal{S}_{t}$ the set of primes $\ell$ satisfying (2) for $f$. We make the following hypothesis:

$\left(H_{p}\right)$ : There exist a square free integer $t$ and a prime number $\ell \in \mathcal{S}_{t}$ such that $\ell=t a^{2}+b^{2}$ and $b$ is a prime number for which $p$ is non-Wieferich.

Theorem 1.2. Let $\mathcal{L}=\left\{\ell_{1}, \ldots, \ell_{s}\right\}$ be a finite set of odd primes. Let $p \geq 5$ be a prime number. Assume that hypotheses $\left(H_{p}\right)$ is satisfied for some prime number $\ell$. Then there is a real quadratic p-rational field $\mathbb{Q}(\sqrt{d})$ for some fundamental discriminant $d<\ell$ such that $\left(\frac{d}{\ell_{k}}\right)=1$ for every $\ell_{k} \in \mathcal{L}$, where $\left(\dot{\overline{\ell_{k}}}\right)$ denotes the Legendre symbol.

\section{2. $p$-RATIONALITY OF QUADRATIC FIELDS}

Let $p$ be an odd prime number and let $\mathcal{O}_{F}^{\prime}=\mathcal{O}_{F}\left[\frac{1}{p}\right]$ be the ring of $p$-integers of $F$, then the field $F$ is called $p$-rational if the étale cohomology group $H^{2}\left(\mathcal{O}_{F}^{\prime}, \mathbb{Z} / p \mathbb{Z}\right)$ vanishies [M-N], [Mo88], [Mo90]. In general, for every integer $i$, if $H^{2}\left(\mathcal{O}_{F}^{\prime}, \mathbb{Z} / p \mathbb{Z}(i)\right)=0$ then we say that the field $F$ is $(p, i)$-regular [A]. If $F$ is totally real, the information about the $p$-rationality and the $(p, i)$-regularity of $F$ are contained in special values of the Dedekind zeta function $\zeta_{F}$ at odd negative integers. More precisely, as a consequence of the Main Conjecture in Iwasawa theory for totally real number fields and odd primes $p$ proved by A.Wiles, we obtain the following case of Lichtenbaum conjecture: for any even positive integer $i \geq 2$, and any totally real number field $F$, we have

$$
w_{i}(F) \zeta_{F}(1-i) \sim_{p}\left|H^{2}\left(\mathcal{O}_{F}^{\prime}, \mathbb{Z}_{p}(i)\right)\right|,
$$

where $w_{i}(F)$ is the order of the group $H^{0}\left(F, \mathbb{Q}_{p} / \mathbb{Z}_{p}(i)\right)$ and $\sim_{p}$ means that they have the same $p$-adic valuation. Moreover, a periodicity statement on cohomology groups gives that

$$
H^{2}\left(\mathcal{O}_{F}^{\prime}, \mathbb{Z} / p \mathbb{Z}(i)\right) \cong H^{2}\left(\mathcal{O}_{F}^{\prime}, \mathbb{Z} / p \mathbb{Z}(j)\right),
$$

whenever $i \equiv j(\bmod p-1)$. Then we have the following proposition:

Proposition 2.1. Assume that $i \geq 2$ is an even integer, then a totally real number field $F$ is $(p, i)$-regular if and only if $w_{i}(F) \zeta_{F}(i)$ is a p-adic unit. 
Proof. The proof follows from (3) and Proposition 2.4 of [A].

Suppose that $F$ is a totally real number field of degree $g$. Let $v_{p}$ be the $p$-adic valuation. We have for even positive integers $i$ the following result [S71, Theorem 6]:

Theorem 2.2. Let $p$ be an odd prime number.

(1) if $g i \equiv 0(\bmod p-1), v_{p}\left(\zeta_{F}(1-i)\right) \geq-1-v_{p}(g)$;

(2) if $g i \not \equiv 0(\bmod p-1), v_{p}\left(\zeta_{F}(1-i)\right) \geq 0$.

In particular, we have

$$
v_{p}\left(\zeta_{F}(2-p)\right) \geq-1-v_{p}(g) .
$$

In [S, Section 3.7], it is suggested that often $v_{p}\left(\zeta_{F}(2-p)\right) \leq-1$. Using Formula (3) we relate the $p$-rationality of $F$ to the special value $\zeta_{F}(2-p)$ in the following way:

Let $p \geq 3$ be a prime which is unramified in $F$, then

$$
F \text { is } p \text {-rational } \Leftrightarrow v_{p}\left(\zeta_{F}(2-p)\right)=-1 \text {. }
$$

For $F=\mathbf{Q}(\sqrt{d})$ a real quadratic fields, the Dedekind zeta function of $F$ satisfies

$$
\zeta_{F}(2-p)=\zeta_{\mathbf{Q}}(2-p) L\left(2-p, \chi_{d}\right) .
$$

Since the field $\mathbf{Q}$ is $p$-rational for every odd prime number $p$ (which is equivalent to say that $\left.v_{p}\left(\zeta_{\mathbf{Q}}(2-p)\right)=-1\right)$, we have the following proposition:

Proposition 2.3. Assume that $p \nmid d$, then the field $\mathbf{Q}(\sqrt{d})$ is p-rational precisely when $v_{p}\left(L\left(2-p, \chi_{d}\right)\right)=0$.

This is the motivation behind using the half-integer weight modular forms called CohenEisenstein series described in the next section.

\section{Cohen-Eisenstein SeRies}

Let $d<0$ be a fundamental discriminant and denote by $h(d)$ the class number of $\mathbf{Q}(\sqrt{d})$. For a rational prime $p \geq 5$, it is known that if $p \nmid h(d)$ then the field $\mathbf{Q}(\sqrt{d})$ is $p$-rational. An object which generate class numbers of imaginary quadratic fields is the 3-power of the standard theta series $\theta$ given by the $q$-expansion

$$
\theta(q)=1+2 q+2 q^{4}+2 q^{9}+\cdots \cdot
$$

This series has been used to prove the existence of infinitely many $p$-rational imaginary quadratic fields. More precisely, the series $\theta^{3}$ is a modular form of weight $\frac{3}{2}$ for the congruence subgroup $\Gamma_{0}(4)$. Write

$$
\theta^{3}(q)=\sum_{n \geq 0} r_{3}(n) q^{n}
$$

then the coefficient $r_{3}(n)$ is the number of times we can write $n$ as a sum of three squares. These coefficients satisfy, by a Theorem of Gauss, the following property:

If $n>3$ is square free, then

$$
r_{3}(n)=\left\{\begin{array}{l}
12 h(-4 n), \quad n \equiv 1,2,5,6 \quad(\bmod 8) \\
12 h(-n), \quad n \equiv 3 \quad(\bmod 8) .
\end{array}\right.
$$

By studying divisibility properties by a prime $p$ of the Fourier coefficients of $\theta^{3}$, one can deduce the existence of infinitely many imaginary quadratic fields $\mathbf{Q}(\sqrt{d})$ with $p \nmid h(d)\left[\mathrm{Ho}_{\mathrm{o}}\right.$, $[\mathrm{Br}]$, hence we have infinitely many $p$-rational imaginary quadratic fields.

To study $p$-rationality of real quadratic fields, we shall use the same approach as for the imaginary quadratic case by considering the following half-integer weight modular forms. 
H. Cohen [C] constructed a modular form $\mathcal{H}_{i}$ of weight $i+\frac{1}{2}$ for the congruence subgroup $\Gamma_{0}(4)$ given by the $q$-expansion:

$$
\mathcal{H}_{i}(q)=\sum_{n \geq 0} h(i, n) q^{n}
$$

such that the Fourier coefficients are essentially given in terms of special values of Dirichlet $L$-functions of quadratic fields:

$$
h(i, n)= \begin{cases}\zeta(1-2 i), & n=0 ; \\ 0, & (-1)^{i} n \equiv 2,3 \quad(\bmod 4) ; \\ L\left(1-i, \chi_{(-1)^{i} d}\right) \sum_{r \mid m} \mu(r) \chi_{(-1)^{i} d}(r) r^{i-1} \sigma_{2 i-1}(m / r), & (-1)^{i} n=d m^{2} .\end{cases}
$$

where $\mu$ is the Möbius function, and $\sigma_{s}(n)$ is the sum of $s$-th powers of the positive divisors of $n$, i.e, $\sigma_{s}(n)=\sum_{r \mid n} r^{s}$.

Let

$$
\epsilon_{d} * \sigma_{2 i-1}(m)=\sum_{r \mid m} \mu(r) \chi_{(-1)^{i} d}(r) r^{i-1} \sigma_{2 i-1}(m / r)
$$

as a convolution product of multiplicative functions, where

$$
\epsilon_{d}(r)=\mu(r) \chi_{(-1)^{i} d}(r) r^{i-1} .
$$

If $d=1$ we write $\epsilon_{d}(n)=\epsilon(n)$; this is a multiplicative function so that

$$
h\left(i, n^{2}\right)=\zeta(1-i) \epsilon * \sigma_{2 i-1}(n) .
$$

Using Proposition 2.3 we see that information about the $p$-rationality of real quadratic fields are encoded in the Fourier coefficients of $\mathcal{H}_{p-1}$. In fact, a field $\mathbf{Q}(\sqrt{d})$ is $p$-rational precisely when

$$
v_{p}(h(p-1, d))=0 .
$$

Based on this observation, the aim is to evaluate the $p$-adic valuation of the coefficients of $\mathcal{H}_{p-1}$. For $p=5$, we shall use the following result of Cohen:

Proposition 3.1. ([C, Proposition 5.1]) Let $r$ be a positive integer. Let $D \equiv 0$ or 1 (mod 4) be an integer such that $(-1)^{r} D=|D|$. Then for $r \geq 2$

$$
\sum_{n \geq 0}\left(\sum_{s} h\left(r, \frac{4 n-s^{2}}{|D|}\right)\right) q^{n} \in M_{r+1}\left(\Gamma_{0}(D), \chi_{D}\right),
$$

where $M_{r+1}\left(\Gamma_{0}(D), \chi_{D}\right)$ is the space of modular forms of weight $r+1$ and level $D$ with character $\chi_{D}$.

As a consequence, Cohen [C, examples, p.277] obtained formulas such as

$$
\sum_{s} h\left(2, N-s^{2}\right)=\frac{-1}{30} \sum_{r \mid N}\left(r^{2}+(N / r)^{2}\right)\left(\frac{-4}{r}\right) .
$$

This equality is used by Beyon [By] to prove the existence of 3-rational real quadratic fields. Using this approach we prove Proposition 4.1 below.

For $p \geq 5$, we use operators introduced by Shimura [Sh] to produce from $\mathcal{H}_{p-1}$ a modular form $G$ with specific Fourier coefficients. For this, let $f=\sum_{n \geq 0} a(n) q^{n}$ be a modular form of weight $i+\frac{1}{2}$ in the space $M_{i+\frac{1}{2}}(N, \chi)$. Let $m>0$ be an integer. The operator $B_{m}$ applies $f$ to the modular form

$$
f \mid B_{m}=\sum_{n \geq 0} a(n m) q^{n m}
$$


which is an element of the space $M_{i+\frac{1}{2}}\left(N m^{2}, \chi\right)$ [Br, Section 3].

The twist operator is defined as follows. Let $\psi$ be a primitive Dirichlet character modulo $m$, then twisting $f$ by $\psi$ gives the modular form

$$
f_{\psi}=\sum_{n \geq 1} \psi(n) a(n) q^{n}
$$

which belongs to the space $M_{i+\frac{1}{2}}\left(N m^{2}, \chi \psi^{2}\right)$ [Sh, Lemma 3.6].

Combining these two operators we obtain the following modular form

$$
\frac{1}{2}\left(f-f \mid B_{m}\right)+\frac{1}{2}\left(f-f \mid B_{m}\right)_{\psi}=\sum_{\psi(n)=1} a(n) q^{n} .
$$

Let $\mathcal{H}$ be the modular form defined by

$$
\mathcal{H}(q)=p\left(\mathcal{H}_{p-1}-\mathcal{H}_{p-1} \mid B_{p}\right)(q)=\sum_{n \geq 1} h_{1}(p-1, n) q^{n}
$$

where $h_{1}(p-1, n)=p h(p-1, n)$. Let $\mathcal{L}=\left\{\ell_{1}, \ldots, \ell_{s}\right\}$ be a set of odd prime numbers and put

$$
\mathcal{N}=\left\{n:\left(\frac{n}{\ell_{k}}\right)=1 \quad \forall \ell_{k} \in \mathcal{L}\right\}
$$

Using (6) for the modular form $\mathcal{H}(q)$ with $m=\ell_{1}$ and $\psi=\left(\dot{\overline{\ell_{1}}}\right)$, we obtain a modular form

$$
G_{1}(q)=\sum_{\left(\frac{n}{\ell_{1}}\right)=1} h_{1}(p-1, n) q^{n} .
$$

Now do the same think for $G_{1}$ and $\ell_{2}$ to obtain a modular form $G_{2}$. By induction on the set $\mathcal{L}$ we obtain the following lemma:

Lemma 3.2. The modular form

$$
G(q)=\sum_{n \in \mathcal{N}} h_{1}(p-1, n) q^{n}
$$

is an element of the space $M_{p-1+\frac{1}{2}}\left(\Gamma_{1}\left(4 p^{2} \prod_{j=1}^{s} \ell_{j}^{4}\right)\right)$.

This modular (with level $\frac{p}{2}$ ) has been used by Ken Ono in [Ono] to study class number of real quadratic fields.

Remark 3.3. (1) Let $n$ be a positive integer such that $\operatorname{gcd}\left(n, \ell_{k}\right)=1$, for every $\ell_{k} \in \mathcal{L}$. Then $n^{2} \in \mathcal{N}$ and the coefficient $h_{1}\left(p-1, n^{2}\right)=\zeta(2-p) \epsilon * \sigma_{2 p-3}(n)$ is a p-adic unit, since $\zeta(2-p)$ has $p$-adic valuation -1 and for every prime number $\ell, \epsilon * \sigma_{2 i-1}\left(\ell^{n}\right) \equiv 1$ $(\bmod p)$. Hence $\mathcal{H}(q)$ is not a constant modulo $p$.

(2) For every integer $n$ such that $h_{1}(p-1, n) \neq 0$, we have $p \nmid n$.

\section{Proof of Theorem 1.1}

In this section, we evaluate sums of Fourier coefficients of the Cohen-Esenstein series $\mathcal{H}_{p-1}$ for $p=5$, to prove the existence of infinitely many 5-rational real quadratic fields.

Proposition 4.1. Let $\ell, \ell^{\prime}$ be distinct odd prime numbers such that $\ell \equiv 1(\bmod 4), \ell^{\prime} \equiv 3$ $(\bmod 4)$ and $v_{5}\left(1-\ell^{\prime 4}\right)=1$. Then there is a positive fundamental discriminant $d_{\ell \ell^{\prime}}<2 \ell \ell^{\prime}$ such that $2 \ell \ell^{\prime}=x^{2}+d_{\ell \ell^{\prime}} y^{2}$ for some integers $x, y$ and $\mathbb{Q}\left(\sqrt{d_{\ell \ell^{\prime}}}\right)$ is a 5-rational number field.

Proof. As a consequence of Proposition 3.1, Cohen [C, page 277] obtained the equality

$$
\sum_{\substack{s \in \mathbf{Z} \\ n-s^{2} \geq 0}} h\left(5-1, n-s^{2}\right)=\frac{1}{300} \sum_{r \mid n}\left(r^{4}+(2 \ell / r)^{4}\right)\left(\frac{-4}{r}\right)+\frac{1}{400} \sum_{n=x^{2}+y^{2}}\left(x^{4}-6 x^{2} y^{2}+y^{4}\right) .
$$


If $n=2 \ell \ell^{\prime}$, where $\ell^{\prime} \equiv 3(\bmod 4)$, then the equation $2 \ell \ell^{\prime}=x^{2}+y^{2}$ has no solutions in integers $x$ and $y$ (it is well known that an integer $n$ is a sum of two squares if and only if all primes $\equiv 3(\bmod 4)$ dividing $n$ have even exponents). Then, in one hand, we have

$$
\frac{1}{400} \sum_{2 \ell \ell^{\prime}=x^{2}+y^{2}}\left(x^{4}-6 x^{2} y^{2}+y^{4}\right)=0 .
$$

In the other hand, the sum

$$
\sum_{s} h\left(5-1, n-s^{2}\right)
$$

is non-empty, since for every integer $m$, the $m$-th coefficient of a Cohen-Eisenstein series $\mathcal{H}_{i}$ is non-trivial if and only if $(-1)^{i} m \equiv 0,1(\bmod 4)$, and for odd integer $x$ such that $2 \ell \ell^{\prime}-x^{2}>0$, we have the congruence $2 \ell \ell^{\prime}-x^{2} \equiv 1(\bmod 4)$.

Moreover, since $\left(\frac{-4}{2}\right)=0$, we have the equality:

$$
\begin{array}{rlc}
\sum_{r \mid 2 \ell \ell^{\prime}}\left(r^{4}+\left(2 \ell \ell^{\prime} / r\right)^{4}\right)\left(\frac{-4}{r}\right) & = & \left(1+\left(2 \ell \ell^{\prime}\right)^{4}\right)+\left(\ell^{4}+\left(2 \ell^{\prime}\right)^{4}\right)-\left(\ell^{\prime 4}+(2 \ell)^{4}\right)-\left(\left(\ell \ell^{\prime}\right)^{4}+2^{4}\right) \\
& = & 1-2^{4}-\left(\ell \ell^{\prime}\right)^{4}\left(1-2^{4}\right)+\ell^{4}\left(1-2^{4}\right)-\ell^{\prime 4}\left(1-2^{4}\right) \\
= & & \left(1-2^{4}\right)\left(1-\left(\ell \ell^{\prime}\right)^{4}+\ell^{4}-\ell^{\prime 4}\right) \\
= & & \left(1+\ell^{4}\right)\left(1-2^{4}\right)\left(1-\ell^{\prime 4}\right) .
\end{array}
$$

Remark that $v_{5}\left(1+\ell^{4}\right)=0, v_{5}\left(1-2^{4}\right)=v_{5}\left(1-\ell^{\prime 4}\right)=1$ and $v_{5}(300)=2$, hence

$$
\frac{1}{300}\left(1+\ell^{4}\right)\left(1-2^{4}\right)\left(1-\ell^{\prime 4}\right) \not \equiv 0 \quad(\bmod 5),
$$

which gives that

$$
\sum_{s} h\left(5-1,6 \ell-x^{2}\right) \not \equiv 0 \quad(\bmod 5) .
$$

Then there is at least one odd integer $x$ such that

$$
v_{5}\left(h\left(5-1,2 \ell \ell^{\prime}-x^{2}\right)\right)=0 .
$$

Since $2 \ell \ell^{\prime}$ is not a sum of two squares, the integer $2 \ell \ell^{\prime}-x^{2}$ is of the form $d_{\ell \ell^{\prime}} y^{2}$, where $d_{\ell \ell^{\prime}}>0$ is a fundamental discriminant. Recall that for every positive integer $i$ and every fundamental discriminant $(-1)^{i} d$ we have

$$
h\left(i, d n^{2}\right)=L\left(1-i, \chi_{\left.(-1)^{i} d\right)}\right) \epsilon_{d} * \sigma_{2 k-1}(n),
$$

and that for a fundamental discriminant $d>0$ and an odd primes $p$ the value $L\left(2-p, \chi_{d}\right)$ is of non-negative $p$-adic valuation [S71, théorème 6]. Then the fundamental discriminant $d_{\ell \ell^{\prime}}$ satisfies:

$$
v_{5}\left(L\left(2-5, \chi_{d_{\ell \ell^{\prime}}}\right)\right)=0 .
$$

Using the equivalence (5) this means that the field $\mathbf{Q}\left(\sqrt{d_{\ell \ell^{\prime}}}\right)$ is 5-rational.

For the proof of Theorem 1.1, we use the following Lemma:

Lemma 4.2. Let $d_{1}, . ., d_{m}$ be positive fundamental discriminants. Then there is a positive density of prime numbers $\ell$ for which the following properties are satisfied:

(1) $\ell \equiv 3(\bmod 4)$

(2) $v_{5}(1-\ell)=1$,

(3) $\left(\frac{d_{i}}{\ell}\right)=1$ for every $i \in\{1, \ldots, m\}$.

Proof. Let consider the following number field $F=\mathbf{Q}\left(\sqrt{d_{1}}, \ldots, \sqrt{d_{m}}, \sqrt{-1}, \mu_{5}, \mu_{25}\right)$, where as usual, $\mu_{n}$ denotes the group of $n$-th root of unity. Since the Galois group $H=\operatorname{Gal}\left(F / \mathbf{Q}\left(\sqrt{d_{1}}, \ldots, \sqrt{d_{m}}\right)\right)$ is cyclic, the Čebotarev's density theorem gives the existence of prime $\ell$ (in fact an infinite numbers of primes) such that the Frobenuis Frob $\mathrm{b}_{\ell}$ at the prime $\ell$ generates the group $H$. In particular these primes $\ell$ satisfy the properties (1), (2) and (3) of the lemma. 
Now we are able to prove Theorem 1.1, which gives the existence of infinitely many 5rational real quadratic fields.

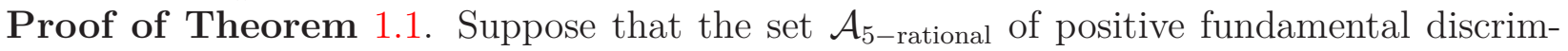
inants $d$ such that $\mathbf{Q}(\sqrt{d})$ is 5-rational is finite. Put $\mathcal{A}_{5 \text {-rational }}=\left\{d_{1}, \ldots, d_{m}\right\}$. Let $\ell$ be a prime number satisfying the assumptions

(1) $\ell \equiv 3(\bmod 4)$

(2) $v_{5}(1-\ell)=1$

(3) $\left(\frac{d_{i}}{\ell}\right)=1$ for every $i \in\{1, \ldots, m\}$.

By Proposition 4.1, for every prime number $\ell^{\prime} \equiv 1(\bmod 4)$, there exists a fundamental discriminant $d_{\ell \ell^{\prime}}<2 \ell \ell^{\prime}$ such that $\mathbf{Q}\left(\sqrt{d_{\ell \ell^{\prime}}}\right)$ is 5-rational, hence there exists $d_{j} \in \mathcal{A}_{5-\text { rational }}$ such that $d_{j}=d_{\ell \ell^{\prime}}$. Moreover, the fundamental discriminant $d_{\ell \ell^{\prime}}$ satisfies the equation $2 \ell \ell^{\prime}=x^{2}+d_{\ell \ell^{\prime}} y^{2}$ for some integers $x, y$, which implies that $\left(\frac{d_{\ell \ell^{\prime}}}{\ell}\right)=\left(\frac{d_{j}}{\ell}\right)=-1$. This is a contradiction since the rational prime $\ell$ satisfy $\left(\frac{d_{k}}{\ell}\right)=1$ for every $k \in\{1, \ldots, m\}$.

\section{Proof of Theorem 1.2}

In this section, we give a sufficient condition (hypothesis $\left(H_{p}^{\prime}\right)$ below) for the existence of real quadratic $p$-rational fields for every prime $p \geq 5$. To do this, we shall use the modular form

$$
G(q)=\sum_{n \in \mathcal{N}} h_{1}(p-1, n) q^{n}
$$

in Lemma 3.2, together with the theta series

$$
\theta_{t}=1+2 \sum_{n \geq 1} q^{t n^{2}}
$$

where $t>1$ is a fundamental disrciminant (cf. [Sh, Section 2] and [S-S, Lemma 2]). Recall that $G$ is an element of the space $M_{p-1+\frac{1}{2}}\left(4 p^{2} \prod_{j=1}^{s} \ell_{j}^{4}, \chi_{0}\right)$, which is a non-constant modular form, where $\mathcal{N}=\left\{n:\left(\frac{n}{\ell_{k}}\right)=1, \forall \ell_{k} \in \mathcal{L}\right\}, \mathcal{L}=\left\{\ell_{1}, \ldots, \ell_{s}\right\}$ is a finite set of odd primes and $\chi_{0}$ is the trivial character. The series $\theta_{t}$ belong to the space $M_{\frac{1}{2}}\left(4 t, \chi_{t}\right)$, where $\chi_{t}$ is the quadratic character associated to the field $\mathbf{Q}(\sqrt{t})$. Then the product $G \theta_{t}$ gives a modular form of weight $p$ in $M_{p}\left(4 t p^{2} \prod_{j=1}^{s} \ell_{j}^{4}, \psi\right)$, where $\psi$ is a Dirichlet character modulo $4 t p^{2} \prod_{j=1}^{s} \ell_{j}^{4}$. We write

$$
G \theta_{t}=\sum_{n \geq 0} c(n) q^{n}
$$

where

$$
c(n)=\sum_{\substack{n=t x^{2}+y \\ y \in \mathcal{N}}} \alpha(x) h_{1}(p-1, y) .
$$

Here $\alpha(x)$ is the $t x^{2}$-th coefficient of $\theta_{t}$.

The result of Serre [S76, page 20], mentioned in the introduction, gives the existence of a set $\mathcal{S}_{t}$ of primes $\ell \equiv 1\left(\bmod 4 t p^{2} \prod_{j=1}^{s} \ell_{j}^{4}\right)$ of positive density such that the congruence $(2)$ holds for $G \theta_{t}$, i.e., for every prime number $\ell \in \mathcal{S}_{t}$ we have:

$$
G \theta_{t} \mid T(\ell) \equiv 2 G \theta_{t} \quad\left(\bmod p^{2}\right)
$$

where

$$
G \theta_{t} \mid T(\ell)=\sum_{n \in \mathcal{N}} c^{\prime}(n) q^{n}
$$

such that $c^{\prime}(n)=c(n \ell)+\psi(\ell) \ell^{p-1} c(n / \ell)$ and $c(n / \ell)=0$ whenever $\operatorname{gcd}(n, \ell)=1$. Hence, for every prime to $\ell$ positive integer $n$, we have

$$
c(n \ell) \equiv 2 c(n) \quad\left(\bmod p^{2}\right)
$$


Recall that $\epsilon_{d} * \sigma_{2 i-1}(m)=\sum_{r \mid m} \mu(r) \chi_{(-1)^{i} d}(r) r^{i-1} \sigma_{2 i-1}(m / r)$. We make the following hypothesis for Serre's primes for the modular form $G \theta_{t}$.

$\left(H_{p}^{\prime}\right)$ : There exist a fundamental discriminant $t$ and a prime number $\ell \in \mathcal{S}_{t}$ such that $\ell=t a^{2}+b^{2}$ and $\epsilon * \sigma_{2 p-3}(b) \not \equiv 1\left(\bmod p^{2}\right)$.

We will see that $\left(H_{p}\right)$ is equivalent to $\left(H_{p}^{\prime}\right)$ if $b$ is a prime number. Under the hypothesis $\left(H_{p}^{\prime}\right)$ we obtain the following result:

Theorem 5.1. Let $\mathcal{L}=\left\{\ell_{1}, \ldots, \ell_{s}\right\}$ be a finite set of odd primes. Let $p \geq 5$ be a prime number. Assume that hypotheses $\left(H_{p}^{\prime}\right)$ is satisfied for some prime number $\ell$. There is a real quadratic p-rational field $\mathbb{Q}(\sqrt{d})$ for some fundamental discriminant $d<\ell$ such that $d \in \mathcal{N}$, which means that $\left(\frac{d}{\ell_{k}}\right)=1$ for every $\ell_{k} \in \mathcal{L}$.

Proof. For every prime number $\ell$ satisfying Serre's congruence for $G \theta_{t}$, we have

$$
c(\ell) \equiv 2 c(1) \quad\left(\bmod p^{2}\right),
$$

where $c(1)=p \zeta(2-p)$ is a $p$-adic unit. Write $c(\ell)$ as follows

$$
c(\ell)=A(\ell)+B(\ell),
$$

where

$$
A(\ell)=\sum_{\substack{\ell=t x^{2}+y \\ y \neq \square \\ y \in \mathcal{N}}} \alpha(x) h_{1}(p-1, y)
$$

and

$$
B(\ell)=\sum_{\substack{\ell=t x^{2}+y^{2} \\ y \in \mathcal{N}}} \alpha(x) h_{1}\left(p-1, y^{2}\right) .
$$

Then we have the following congruence

$$
A(\ell) \equiv 2 c(1)-B(\ell) \quad\left(\bmod p^{2}\right) .
$$

Moreover, $\ell \equiv 1(\bmod 4 t)$ implies that the equation

$$
\ell=t x^{2}+y^{2}
$$

has a unique solution $(a, b)$, see e.g., [Co, Chapter 1, page 31]. Hence the sum $B(\ell)$ is non-trivial and

$$
B(\ell)=2 p \zeta(2-p) \epsilon * \sigma_{2 p-3}(b) .
$$

We obtain the congruence

$$
A(\ell) \equiv 2 p \zeta(2-p)\left(1-\epsilon * \sigma_{2 p-3}(b)\right) \quad\left(\bmod p^{2}\right) .
$$

Since hypotheses $\left(H_{p}^{\prime}\right)$ is satisfied for the prime number $p$, there exist a square free integer $t$ and a prime number $\ell \in \mathcal{S}_{t}$ such that $\ell=t a^{2}+b^{2}$, with $b$ satisfying the property

$$
\epsilon * \sigma_{2 p-3}(b) \not \equiv 1 \quad\left(\bmod p^{2}\right) .
$$

This leads to the property

$$
A(\ell) \not \equiv 0 \quad\left(\bmod p^{2}\right) .
$$

Moreover, we have by construction

$$
A(\ell)=\sum_{\substack{\ell=t x^{2}+y \\ y \neq \square}} a(x) h_{1}(p-1, y)
$$


such that each component of $A(\ell)$ is of $p$-adic valuation $\geq 1$. By (10) there exist integers $x$ and $y$ such that $\ell=t x^{2}+y, y$ is not a square and $v_{p}\left(\alpha(x) h_{1}(p-1, y)\right)=1$. Hence by definition of $h(p-1, y)$, there exists a fundamental discriminant $d<\ell$ such that $y=d y_{1}^{2}$ and $v_{p}\left(L\left(2-p, \chi_{d}\right)\right)=0$. Then Proposition 2.3 and the second statement of remark 3.2, gives that the field $\mathbb{Q}(\sqrt{d})$ is $p$-rational and satisfies the decomposition conditions.

The statement of Hypothesis $\left(H_{p}\right)$ in the introduction involves the so called Wieferich primes defined as follows:

Definition 5.2. Let $a>1$ be an integer. A prime number $p \nmid a$ is said to be a Wieferich prime of basis a if

$$
a^{p-1}-1 \equiv 0 \quad\left(\bmod p^{2}\right) .
$$

Otherwise, the prime $p$ is said to be non-Wieferich.

Let $b=p_{1}^{n_{1}} \ldots p_{s}^{n_{s}}$ be an integer. Remark that

$$
\epsilon * \sigma_{2 p-3}(b)=\prod_{i=1}^{s} \epsilon * \sigma_{2 p-3}\left(p_{i}^{n_{i}}\right),
$$

and

$$
\epsilon * \sigma_{2 p-3}\left(p_{i}^{n_{i}}\right)=1+p_{i}^{p-2} \sigma_{2 p-3}\left(p_{i}^{n_{i}-1}\right)\left(p_{i}^{p-1}-1\right) .
$$

Suppose that the integer $b$ is a prime number, then

$$
\epsilon * \sigma_{2 p-3}(b)=1+b^{p-2}\left(b^{p-1}-1\right) .
$$

Hence $\epsilon * \sigma_{2 p-3}(b) \not \equiv 1\left(\bmod p^{2}\right)$ precisely when $p$ is a non-Wieferich prime of basis $b$. Hence $\left(H_{p}\right)$ is equivalent to $\left(H_{p}^{\prime}\right)$ in this case and Theorem 1.2 is proved.

Remark 5.3. Let $i$ be an even integer and let $\mathcal{H}_{i}$ be the Cohen-Eisenstein series of weight $i+\frac{1}{2}$. Under adequate hypotheses $\left(H_{(p, i)}\right)$, analogous arguments gives the existence of real quadratic $(p, i)$-regular number fields with prescribed arithmetic properties as in Theorem 5.1.

\section{REFERENCES}

[A] Assim, J. Codescente en K-théorie étale et de nombres, manuscripta math. 86, (1995), 499-518.

[Br] Bruinier, J.H. Nonvanishing modulo $\ell$ of Fourier coeffients of half-integral weight modular forms, Duke Math. J. 98 (1999), 595-611.

[By] Byeon, D. Indivisibility of special values of Dedekind zeta functions of real quadratic fields, Acta Arithmetica. 2003.

[C] Cohen, H. Sums involving the values at negative integers of L-functions of quadratic characters, Math. Ann. 217 (1975), 271-285.

[Co] Cox, D. A. Primes of the form $x^{2}+n y^{2}$ : Fermat, class field theory, and complex multiplication. Vol. 34. John Wiley and Sons, (2011).

[Ha] Hartung, P. Proof of the existence of infinitely many imaginary quadratic fields whose class number is not divisible by 3, J. Number Theory 6 (1974) 276-278

[Ho] Horie, K. Trace formulae and imaginary quadratic fields, Math. Ann (4) 288 (1990) 605-612.

[G] Greenberg, R. Galois representations with open image, Annales mathématiques du Québec, 40 (2016), 83-119.

[Ko] Koblitz, N. Introduction elliptic curves and modular forms, Springer Verlag, 1984.

[Kol] Kolster, M. K-theory and arithmetic, No. INIS-XA-986. 2003.

[M-N] Movahhedi, A and Nguyen, Q.D.Sur l'arithmétique des corps de nombres p-rationnels, Sém de théo des nombres de Paris, 1987-1988, Bikhäuser, Vol 81, (1990) 155-200.

[Mo88] Movahhedi, A. Sur les p-extensions des corps p-rationnels, These Paris VII (1988).

[Mo90] Movahhedi, A. Sur les p-extensions des corps p-rationnels, Math. Nach, 149, (1990), 163-176.

[Ono] Ono, K. indivisibility of class numbers of real quadartic fields, compositio math, 119 (1999), 1-11.

[S] Serre, J.-P. Cohomologie des groupes discrets, Annals of Mathematics Studies 70, Princeton University Press, 1971.

[S71] Serre, J.-P. Congruences et formes modulaires, seminaire N.Bourbaki, 1971-1972, p.319-338.

[S73] Serre, J.-P. A course in arithmetic, Springer Verlag 1973. 
[S76] Serre, J.-P. Divisibilité de certaines fonctions arithmétiques, L'Ensein. Math. 22 (1976), 227- 260.

[S-S] Serre, J.-P., and Stark, H. M. Modular forms of weight 1/2. In Modular functions of one variable VI (pp. 27-67). Springer, Berlin, Heidelberg, (1977).

[Sh] Shimura, G. On modular forms of half integral weight. Ann. of math. 97, (1973), 440-481.

[Wa] Washington, L. Introduction to Cyclotomic Fields(2nd ed.), Graduate Texts in Math. 83, SpringerVerlag (1997).

[W] Wiles, A. On class group of imaginary quadratic fields, J.London Math. soc. (2) 92 (2015), 411-426.

(1) Moulay Ismail University, Department of mathematics, Faculty of sciences, Meknès, B.P. 11201 Zitoune, Meknès, Morocco.

E-mail address: j.assim@yahoo.fr

(2) Moulay Ismail University, Department of mathematics, Faculty of sciences, Meknès, B.P. 11201 Zitoune, Meknès, Morocco.

E-mail address: z.bouazzaoui@edu.umi.ac.ma 